\title{
Knockdown of PKM2 enhances radiosensitivity of cervical cancer cells
}

\author{
Yanzhu Lin ${ }^{1+}$, Hui Zhai ${ }^{2+}$, Yi Ouyang ${ }^{1+}$, Zhiyuan $\mathrm{Lu}^{3}$, Chengbiao $\mathrm{Chu}^{4}$, Qianting $\mathrm{He}^{3}$ and Xinping Cao ${ }^{{ }^{*}}$
}

\begin{abstract}
Background: Pyruvate kinase isozyme type M2 (PKM2) catalyzes the final step in glycolysis and has been found to be up-regulated in multiple human malignancies. However, whether PKM2 regulates the radiosensitivity of human cervical cancer (CC) remains unknown.

Methods: The expression of PKM2 in 94 patients with CC in the complete response (CR) and noncomplete response (nCR) groups, was evaluated by immunohistochemistry. The effect of PKM2 inhibition on radiosensitivity, the cell cycle, DNA damage, and apoptosis was evaluated by immunofluorescence analysis, colony formation assay, flow cytometry analysis and Western blotting.
\end{abstract}

Results: PKM2 expression was more highly expressed in the $\mathrm{nCR}$ group than that in CR group and PKM2 expression was enhanced in CC cells after ionizing radiation (IR). In addition, knockdown of PKM2 combined with IR significantly reduced cell growth, promoted apoptosis, and enhanced radiosensitivity. Additionally, knockdown of PKM2 with IR resulted in increased phosphorylation of DNA repair checkpoint proteins (ATM) and phosphorylated-H2AX. Moreover, knockdown of PKM2 combined with IR significantly increased the expression of cleaved caspase 3 and caspase 9, whereas Bcl2 expression was suppressed. Furthermore, knockdown of PKM2 combined with IR markedly reduced the expression of several cancer stem cell biomarkers in vitro, including NANOG, OCT4, SOX2, and Bmi1.

Conclusions: The results of our study suggests that PKM2 might be involved in mediating CC radiosensitivity and is identified as a potentially important target to enhance radiosensitivity in patients with CC.

Keywords: Radiosensitivity, Apoptosis, Cell cycle, Double strand break repair

\section{Background}

Cervical cancer $(\mathrm{CC})$ is the second most common gynecological cancer worldwide and seriously threatens women's health [1]. Radiotherapy is one of the most effective modalities for the treatment of locoregionally advanced CC (LACC). Unfortunately, in some patients, cancer cells acquire radioresistance during radiotherapy, leading to local therapeutic failure, which is responsible for the high recurrence rate and poor survival among patients with CC [2]. Therefore, novel strategies intended to enhance the radiosensitivity of cancer cells

\footnotetext{
*Correspondence: caoxp@sysucc.org.cn

${ }^{\dagger}$ Yanzhu Lin, Hui Zhai and Yi Ouyang contributed equally to this work

1 Department of Radiation Oncology, Sun Yat-sen University Cancer Center, State Key Laboratory of Oncology in South China, Collaborative Innovation Center for Cancer Medicine, Guangzhou, China Full list of author information is available at the end of the article
}

and research on the underlying mechanisms of $\mathrm{CC}$ are essential.

Previous studies have shown that the high aerobic glycolysis rate of malignant tumors results in higher lactic acid production, which is closely related to metastasis and radioresistance of cancers [3, 4]. Additionally, other studies have found that inhibition of glycolysis increases radiosensitivity [5-7]. However, the mechanism through which inhibition of glycolysis increases radiosensitivity remains poorly understood. Pyruvate kinase isozyme type M2 (PKM2) is a rate-limiting enzyme in the final step of the glycolytic pathway, which catalyzes the conversion of phosphoenolpyruvate (PEP) and adenosine diphosphate (ADP) to pyruvate and is responsible for ATP production $[8,9]$. Meng et al. [10] found that knockdown of PKM2 expression enhances the radiosensitivity of non-small-cell lung 
cancer cell lines through inhibition of AKT and PDK1 phosphorylation and increases the rate of ERK1/2 and GSK3 $\beta$ phosphorylation. Another study showed that overexpression of PKM2 is associated with radiation resistance in CC, but its molecular mechanism was not established [11]. We previously reported that PKM2 was up-regulated in $\mathrm{CC}$ and may serve as a molecular target [12]. However, the role of PKM2 in radiosensitivity of CC has not been elucidated.

It has been demonstrated that the presence of cancer stem cells (CSCs) in solid tumors is a major factor in radioresistance [13]. Treatment modalities targeting CSCs may substantially improve outcome in patients with cancer. Accordingly, recent studies have increasingly focused on the identification of CSC-specific markers, such as CD44, CD133, ABCG2 (CD338), and ALDH1 and stem cell transcription factors SOX2, OCT4, and NANOG. However, the molecular mechanism of radioresistance in CSCs remains unclear. A previous study showed that nuclear PKM2 expression positively correlates with stemcell-like properties [14]. PKM2 has also recently been found to regulate gene transcription of OCT4 $[15,16]$. Therefore, the role of PKM2 in cervical CSCs requires further exploration.

The main goal of this study was to examine the expression of PKM2 in association with radiation resistance in $\mathrm{CC}$ and to investigate the radiotherapy resistance mechanism at the molecular level.

\section{Materials and methods Sample collection}

A total of 94 patients who had been treated with definitive radiotherapy for CC at Sun Yat-sen University Cancer Center (Guangzhou, China) from November 2016 to December 2017 were analyzed. The collection of human tissue samples was approved and supervised by the Ethics Committee of Sun Yat-sen University. All specimens had confirmed pathological diagnoses. Patients with distant metastasis were excluded. The patients were treated with definitive radiotherapy consisting of external beam radiotherapy (intensity-modulated radiotherapy) followed by high-dose-rate brachytherapy with or without platinumbased concurrent chemotherapy. Immunohistochemistry (IHC) was performed as previously described [12]. The proportion of PKM2 staining was graded as $(1-25 \%=1$; $26-50 \%=2 ; 51-75 \%=3 ;>76 \%=4)$ and the staining intensity was graded as (negative $=0$; weak $=1$; moderate $=2$; strong $=3$ ). The final score for PKM2 expression was calculated by multiplication of these 2 scores. Slides were considered low or high, with scores of $\leq 7$ or $>7$, respectively.

\section{Response evaluation}

The response to treatment was assessed according to the Response Evaluation Criteria in Solid Tumors after the completion of radiotherapy [17]. A complete response (CR) was defined as the disappearance of all target and nontarget lesions and no new lesions documented after two assessments at least 4 weeks apart. A partial response was defined as the detection of at least a $30 \%$ reduction in the sum of the longest dimensions of the target lesions in two assessments at least 4 weeks apart. Progressive disease was defined as a $20 \%$ increase in the sum of the longest dimensions of the target lesions or the development of new lesions. Stable disease implies that none of the previously mentioned statuses applied. At 3 months after treatment, all patients were divided into two groups: $\mathrm{CR}$ and noncomplete response (nCR) groups.

\section{Cell culture}

The CC cell lines SiHa and HeLa were obtained from the American Type Culture Collection (Manassas, VA, United States) and were cultured in DMEM (Invitrogen, Carlsbad, CA, United States) or 1640 (Gibco; Gaithersburg, MD, United States) containing 10\% fetal bovine serum, $100 \mu / \mathrm{mL}$ penicillin, and $100 \mu \mathrm{g} / \mathrm{mL}$ streptomycin at $37^{\circ} \mathrm{C}$ in $5 \%$ humidified $\mathrm{CO}_{2}$. Stable knockdown PKM2 $\mathrm{SiHa}$ and HeLa cell lines were constructed as previously described [12].

\section{Cell viability assay}

HeLa and SiHa cells were seeded at a density of $10^{4}$ cells/ $\mathrm{mL}$ in 96-well plates and incubated at $37{ }^{\circ} \mathrm{C}$ overnight. Cells were treated with various doses of ionizing radiation (IR) (0, 2, 4, 6, and 8 Gy) using an RS2000 X-ray irradiator (Rad Source; Rad Source Technologies, Coral Springs, FL, United States) at a dose rate of $1.1 \mathrm{~Gy} / \mathrm{min}$. After treatment, the cells were incubated for an additional $48 \mathrm{~h}$, and cell viability was determined using Cell Counting Kit-8 (Dojindo; Kumamoto, Japan) and by measuring absorbance at $450 \mathrm{~nm}$ using an ELx800 ${ }^{\mathrm{TM}}$ plate reader (BioTek; Winooski, VT, USA) following the manufacturer's instructions.

\section{Clonogenic cell survival assay for cell survival fraction analysis}

Radioresponse was assessed using a colony formation assay. Different numbers of cells were seeded into sixwell plates $(150,300,600,1000$, and 2000 per well). The next day, cells were irradiated with $0,2,4,6$, and 8 Gy by an X-ray irradiator at room temperature. After IR, cells were incubated for 14 days to form colonies. Colonies were washed twice with PBS, fixed with methanol, and stained with crystal violet; colonies of more than 50 cells 
were then counted. Plating efficiency (PE) was calculated in triplicate as $\mathrm{PE}=$ (colony number/plating cell number) $\times 100 \%$. The surviving fraction $(\mathrm{SF})$ was estimated by calculating $\mathrm{SF}=$ colony number $/$ (cells seeded $\times$ plating efficiency).

\section{Western blot}

Total proteins were extracted as described previously [12]. Cells were lysed in radioimmunoprecipitation assay buffer (Beyotime; Haimen, China), and protein concentrations were measured through bicinchoninic protein assay kit (Pierce, Appleton, WI, USA). Equal amounts of protein were separated through SDS-PAGE and subsequently transferred to a PVDF membrane (Millipore; Burlington, MA, United States). After the membrane was blocked for $1 \mathrm{~h}$ using 5\% skim milk, it was incubated with primary antibody overnight at $4{ }^{\circ} \mathrm{C}$. Finally, the membrane was incubated with respective secondary antibodies (Santa Cruz, United States) for $1 \mathrm{~h}$. Signals were detected using enhanced chemiluminescence reagents (Pierce; Waltham, MA, United States). Sources of antibodies and concentrations used were as follows: rabbit anti-PKM2 (1:1000, CST, USA), rabbit anti-phospho-ATM (Ser1981) (1:1000, CST, USA), rabbit antiphospho-BRCA1 (Ser1524) (1:1000, CST, USA), rabbit anti-phospho-Chk1 (Ser345) (1:1000, CST, USA), rabbit anti-phospho-Chk2 (Thr68) (1:1000, CST, USA), rabbit anti-phospho-histone H2AX (Ser139) (1:1000, CST, USA), mouse anti-cycline B1 (1:1000, Santa Cruz, USA), mouse anti-phospho-p53 (Ser15) (1:1000, CST, USA), rabbit anti-cleaved caspase 3 (1:1000, CST, USA), rabbit anti-caspase 3 (1:1000, CST, USA), rabbit anti-cleaved caspase 9 (1:1000, CST, USA), mouse anti-caspase 9 (1:1000, CST, USA), rabbit anti-Bcl2 (1:1000, CST, USA), rabbit anti-Bax (1:1000, CST, USA), rabbit anti-NANOG (1:1000, Abcam, USA), rabbit anti-OCT4 (1:1000, CST, USA), rabbit anti-SOX2 (1:1000, CST, USA), rabbit antiKLF4 (1:1000, CST, USA), mouse anti-ABCG2 (1:1000, CST, USA), mouse anti-Bmi1 (1:1000, Santa Cruz, USA), rabbit anti-GAPDH (1:1000, CST, USA). Glyceraldehyde 3-phosphate dehydrogenase (GAPDH) was used as the loading control. Quantity One Software (Bio-Rad) was used to analyze the intensity of blots.

\section{RNA extraction and quantitative RT-PCR}

Total RNA was extracted from the HeLa and SiHa cells using TRIzol (Invitrogen; Milan, Italy) according to manufacturer's protocol, and $500 \mathrm{ng}$ was used to obtain cDNA through reverse transcription using PrimeScript RTMasterMix (Promega; Madison, WI, United States). Quantitative RT-PCR was conducted using an SYBR Green PCR master mix (Roche; Basel, Switzerland) on the CFX96 Real-Time PCR Detection System (Bio-Rad;
Hercules, CA, United States). PCR amplification was performed with the specific primer sets as described previously [12]. Relative expression was normalized to the expression of $\beta$-actin. The $2^{-\Delta \Delta \mathrm{Ct}}$ method was used for relative quantification of gene expression. The primers used in the studies were: PKM2 sense, $5^{\prime}$-ATGTCGAAG CCCCATAGTGAA- $3^{\prime}$, PKM2 antisense, $5^{\prime}$-TGGGTG GTGAATCAATGTCCA- $3^{\prime}, \beta$-actin sense, $5^{\prime}$-CTCCAT CCTGGCCTCGCTGT-3', $\beta$-actin antisense, $5^{\prime}$-GCT GTCACCTTCACCGTTCC-3'.

\section{Cell cycle analysis}

$\mathrm{HeLa}$ and $\mathrm{SiHa}$ cells that were irradiated with 2 Gy were collected at $24 \mathrm{~h}$ after IR and assessed for cell cycle distribution through flow cytometry. Briefly, $10^{6}$ cells were harvested with trypsin and fixed in $70 \%$ ethanol for $24 \mathrm{~h}$. The cells were then washed and suspended in $500 \mu \mathrm{L}$ of PBS buffer containing $25 \mathrm{mg} / \mathrm{mL}$ RNAse and $50 \mu \mathrm{g} /$ $\mathrm{mL}$ propidium iodide and incubated for $15 \mathrm{~min}$ in the dark. The cells were subsequently detected through flow cytometry (Beckman Coulter, Inc.; Brea, CA, US), and the data were analyzed by Modifit (Beckman Coulter).

\section{Apoptotic assays}

Apoptotic cell death was assessed through flow cytometry using the Annexin V-APC/7-AAD Apoptosis Detection Kit (BestBio; Shanghai, China) according to the manufacturer's instructions. Briefly, the indicated cells were exposed to 0 or 2 Gy of IR. After treatment, cells were incubated at $37{ }^{\circ} \mathrm{C}$ for $48 \mathrm{~h}$. Then, $10^{5}$ cells were resuspended in $500 \mu \mathrm{L}$ of $1 \times$ binding buffer and stained with $10 \mu \mathrm{L}$ of Annexin V-APC and $5 \mu \mathrm{L}$ of 7-AAD. Apoptosis levels were detected through flow cytometry (Beckman Coulter).

\section{Immunofluorescence}

Immunofluorescence detection of phospho-histone$\mathrm{H} 2 \mathrm{AX}(\gamma-\mathrm{H} 2 \mathrm{AX})$ foci was performed to monitor formation of double-strand DNA breaks (DSBs). At specified time points $(1,12$, and $24 \mathrm{~h})$ after treatment with $2 \mathrm{~Gy}$, cells were fixed with $4 \%$ paraformaldehyde for $15 \mathrm{~min}$, permeabilized with $0.2 \%$ Triton X-100 for 5 min, blocked with $5 \%$ bovine serum albumin in PBS, and incubated overnight at $4{ }^{\circ} \mathrm{C}$ with $\gamma$-H2AX (Ser139, 1:1000, CST) antibody. For visualization, cells were incubated with Alexa Fluor 488 conjugated goat antirabbit secondary antibody (Abcam; Cambridge, UK; 1:1000) for $1 \mathrm{~h}$. DAPI (Sigma-Aldrich; St. Louis, MO, United States; 1:3000) was used as a nuclear counterstain. Foci of $\gamma-\mathrm{H} 2 \mathrm{AX}$ were counted from at least five random fields under a Leica Confocal Laser Scanning Microscope (Leica Microsystems; Wetzlar, Germany). 


\section{Statistical analysis}

Each experiment was performed in triplicate. Data are expressed as mean \pm SD (standard deviation). The statistical analysis was performed using SPSS 20.0 (SPSS, Inc.; Chicago, IL, United States). The significance of differences between two groups was determined by the t-test and One-way analysis of variance was used for multiple comparisons. $P<0.05$ was defined as statistically significant.

\section{Results}

\section{PKM2 is involved in tumor radiosensitivity}

PKM2 expression in CC tissues was detected by IHC staining. In total, $36(38 \%)$ patients had CR, and 58 patients (62\%) had nCR (Table 1). The results revealed that the PKM2 expression in the radiation-resistant group was statistically significantly higher than that in the radiation-sensitive group (Fig. 1a, b, Table 1, $P=0.002$ ). However, there were no statistically significant differences between the radiation response and the clinicopathological features (Additional file 1: Table S1). These results suggested that PKM2 is clinically associated with resistance to radiation.

To test this hypothesis, we first treated HeLa and SiHa cells with increasing doses of IR. Then, we showed, by Western blot and quantitative RT-PCR analyses, that the expressions of PKM2 increased after exposure to different radiation doses in cell lines (Fig. 1c, d), suggesting that PKM2 might potentially play a role in modulating radiosensitivity of CC cells.

\section{Knockdown of PKM2 enhanced cell radiosensitivity}

We also examined the role of PKM2 in radioresponsiveness in HeLa and SiHa cells. Stable low PKM2 expression in HeLa and $\mathrm{SiHa}$ cell lines was established using a lentiviral expression system as previously reported [12]. The results of the cell viability assay showed that the viability of PKM2 knockdown cells was significantly lower than that of control cells after exposure to various doses of IR (Fig. 2a, b). To further validate the effect of PKM2 silencing on radiosensitivity, colony formation ability was assayed after IR exposure. The number of colonies formed by PKM2-shRNA cells was significantly

\begin{tabular}{|c|c|c|c|c|}
\hline \multirow[t]{2}{*}{ Group } & \multirow{2}{*}{$\begin{array}{l}\text { Patients } \\
(n=94)\end{array}$} & \multicolumn{2}{|c|}{ PKM2 expression } & \multirow[t]{2}{*}{$P$-value } \\
\hline & & $\begin{array}{l}\text { Low- } \\
\text { expression } \\
(n=46)\end{array}$ & $\begin{array}{l}\text { High- } \\
\text { expression } \\
(n=48)\end{array}$ & \\
\hline Radiation-sensitive & 36 & 25 & 11 & 0.002 \\
\hline Radiation-resistant & 58 & 21 & 37 & \\
\hline
\end{tabular}

decreased compared with that of control cells (Fig. 2c, d). These results suggested that knockdown of PKM2 made cells more sensitive to IR compared with the control cells. Similar results were obtained with $\mathrm{SiHa}$ cells. SF curves also revealed that the clonogenicity of PKM2-shRNA group cells was dramatically reduced in an ionizing radiation-dose-dependent manner (Fig. 2e, f). These results suggested that PKM2 inhibition can enhance radiosensitivity in CC cells.

\section{Knockdown of PKM2 increased DNA damage}

The phosphorylated form of $\mathrm{H} 2 \mathrm{AX}, \gamma-\mathrm{H} 2 \mathrm{AX}$, has been identified as a marker of the early response to DNA damage [18]. To determine whether knockdown of PKM2 regulates radiation-induced DNA DSBs, the number of $\gamma$-H2AX foci at different times after IR (2 Gy) was counted. As shown in Fig. 3, the number of $\gamma-\mathrm{H} 2 \mathrm{AX}$ foci in the control group increased at $1 \mathrm{~h}$ after IR, and decreased rapidly at $24 \mathrm{~h}$ after IR. In contrast to the control group, number of $\gamma-\mathrm{H} 2 \mathrm{AX}$ foci decreased more slowly in the PKM2 silencing group (Fig. 3a, b). The data revealed that knockdown of PKM2 increased DNA damage in response to ionizing radiation treatment.

\section{Knockdown of PKM2 promoted radiosensitivity by arresting cells in the cell cycle G2/M phase and inducing apoptosis}

The effect of PKM2 inhibition on cell cycle distribution was investigated. The cell cycle distribution of HeLa and $\mathrm{SiHa}$ cells revealed that PKM2 knockdown significantly increased the proportion of cells in the G2/M phase (Fig. 4a and Additional file 2: Figure S1A and C). We also analyzed the role of PKM2 in the cell cycle in response to radiation. The proportion of the shPKM2+ radiation group cells in the G2/M phase was significantly higher compared with that of the control group in HeLa and SiHa cells (Fig. 4c and Additional file 2: Figure S1A and C). The data suggest that knockdown of PKM2 induced an accumulation of cells, indicative of a G2/M arrest after IR.

We analyzed the effect of PKM2 knockdown on IRinduced cell apoptosis. The apoptosis levels were significantly increased in the HeLa PKM2-shRNA groups (Fig. 4b). In addition, a significantly increased apoptosis rate was observed in HeLa PKM2-shRNA cells after IR compared with the control group. This indicates that PKM2 inhibition enhances radiation-induced apoptosis. Additionally, knockdown of PKM2 in SiHa cells significantly enhanced radiation-induced cell apoptosis (Additional file 2: Figure S1B and D). According to these findings, knockdown of PKM2 promoted radiosensitivity by enhancing IR-induced apoptosis of CC cells. 
a

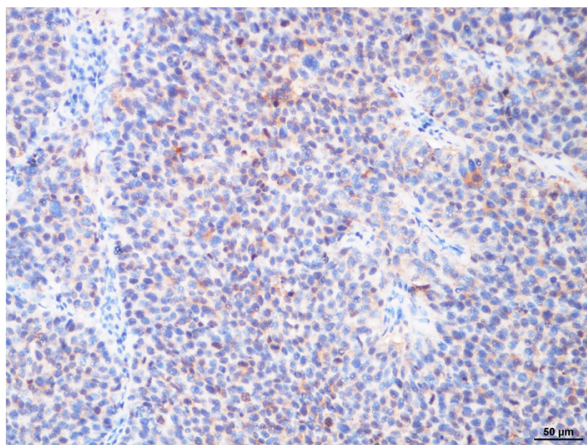

C

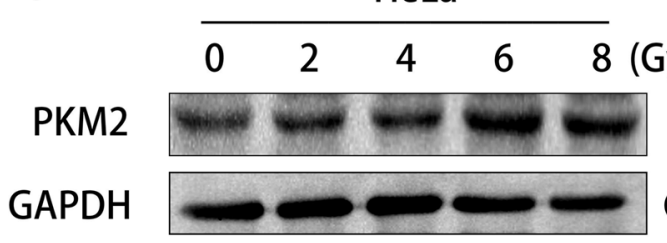

e

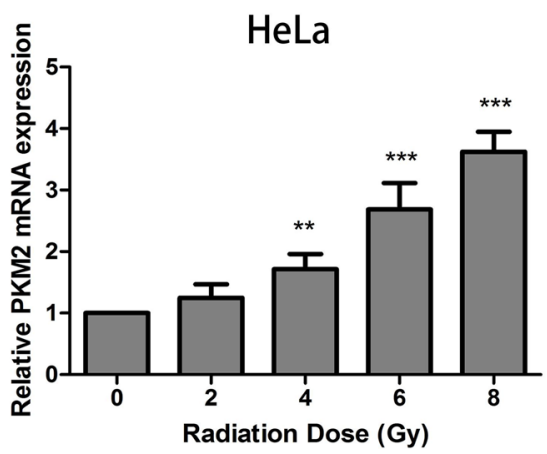

APDH b

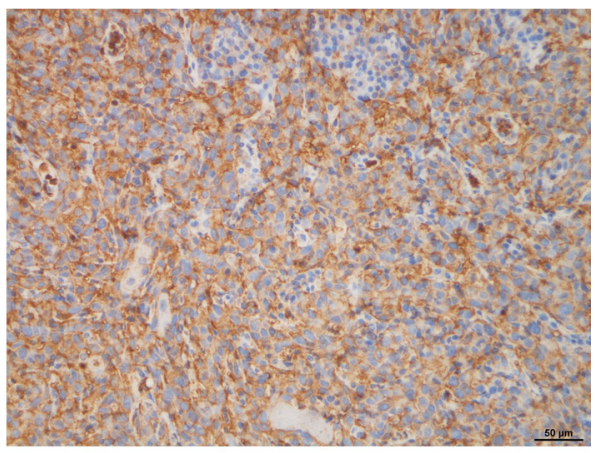

d

$\mathrm{SiHa}$

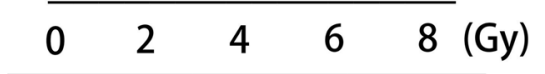

$\begin{array}{llllll}0 & 2 & 4 & 6 & 8 & \text { (Gy) }\end{array}$

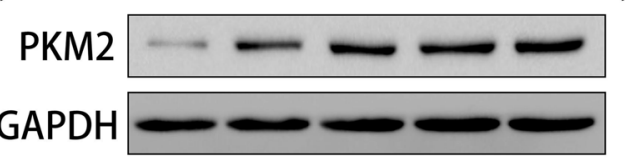

f

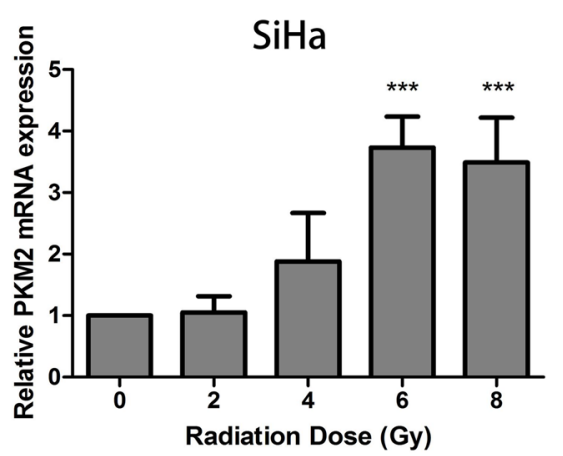

Fig. 1 PKM2 expression was associated with radiation resistance. Representative examples of PKM2 staining of cervical cancers in the radiation-resistant group and radiation-sensitive group. a Weak positive staining of PKM2 in the radiation-sensitive group. $\mathbf{b}$ Strong positive staining of PKM2 in the radiation-resistant group. $\mathbf{c}$, $\mathbf{d}$ HeLa and SiHa cells were cultured for $24 \mathrm{~h}$ after $0,2,4,6$, and 8 Gy of X-ray irradiation. Total protein were extracted and analyzed through Western blot. e, f HeLa and SiHa cells were cultured for 24 h after 0, 2, 4, 6, and 8 Gy of X-ray irradiation. Total RNA were extracted and analyzed through quantitative RT-PCR. Data represent three independent experiments. ${ }^{* *} P<0.01,{ }^{* * *} P<0.001$ compared with the 0 Gy group

We additionally evaluated the levels of cell cycle regulatory protein to determine the effect of PKM2 on radiationinduced DNA damage and repair. As shown in Fig. 4e, in HeLa cells, the levels of the p-ATM, p-Chk1, and p-Chk2 proteins, which are key checkpoint proteins, are higher in sh-PKM2 cells than in control cells, whereas cyclin B1 levels are decreased. Also, knockdown of PKM2 in HeLa cells led to upregulation of phospho-ATM, Chk1, p53, and $\gamma-\mathrm{H} 2 \mathrm{AX}$, after IR treatment. These findings indicated that the DNA damage checkpoint response was activated.

\section{Knockdown of PKM2 reduced CSC transcription factors after IR}

To elucidate the molecular mechanism of PKM2-mediated radiosensitivity of $\mathrm{CC}$ cells, the levels of several apoptosis-related proteins were measured in cells following radiation treatment. As shown in Fig. 5a, PKM2 inhibition increased the expression of cleaved caspase 3 and cleaved caspase 9 and reduced $\mathrm{Bcl} 2$ expression in irradiated HeLa cells (Fig. 5a), which contribute to apoptosis after IR.

Since CSCs and radioresistance are related, we also evaluated the effect of PKM2 silencing on the expression of stemness-associated transcription factors (KLF4, SOX2, OCT4, ABCG2, and NANOG). The results showed a drastic decrease in the levels of SOX2, OCT4, ABCG2, Bmi1, and NANOG in shPKM2 HeLa cells compared with those in the control HeLa cells. Additionally, the expression levels of SOX2, OCT4, ABCG2, Bmi1, and NANOG in shPKM2 HeLa 

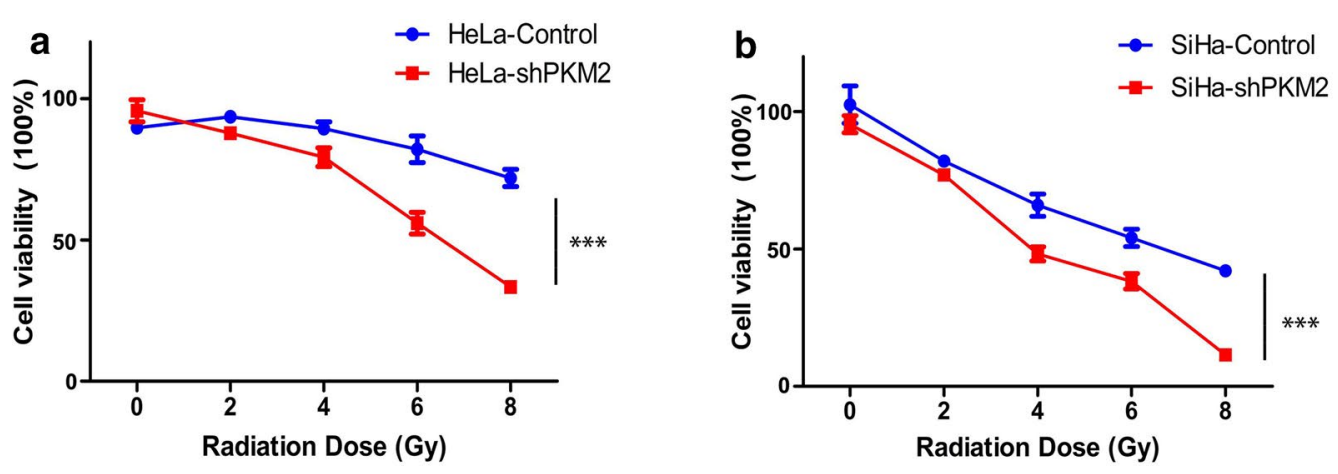

C
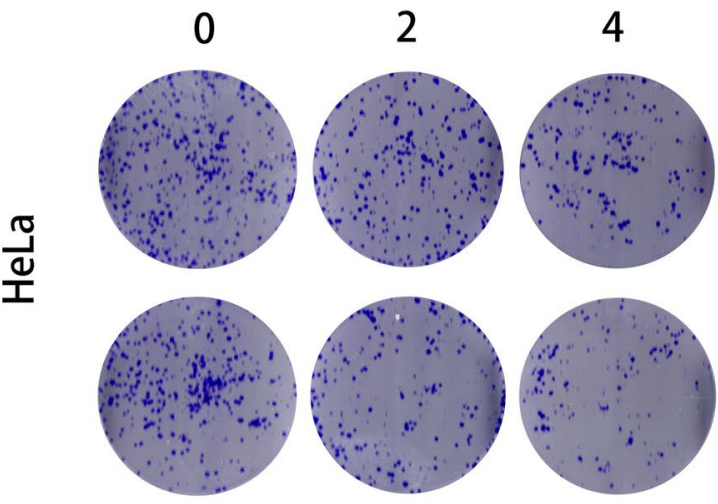

6

8 (Gy)

d

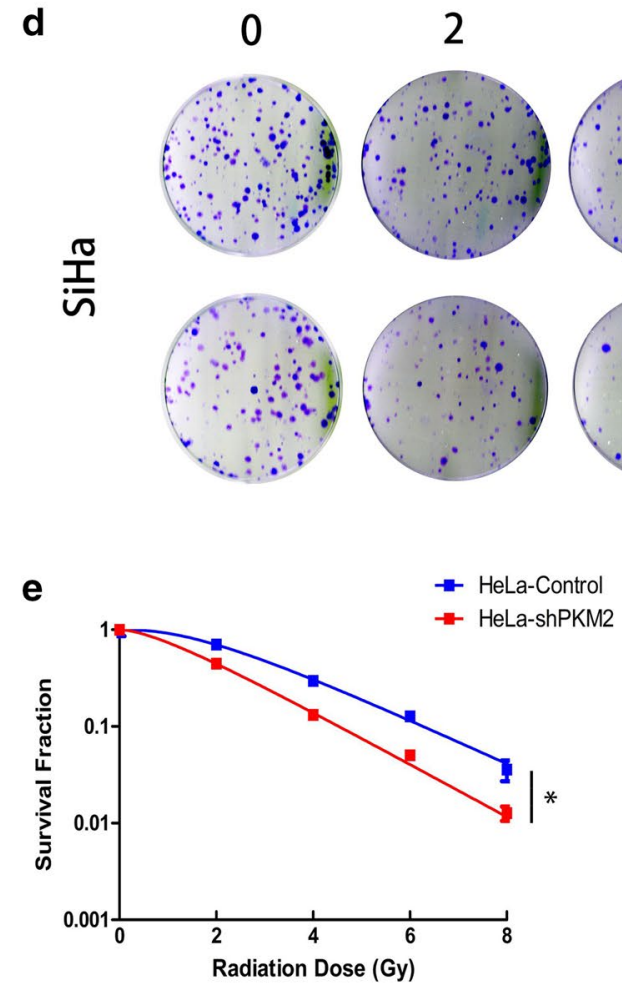

4

6

8 (Gy)
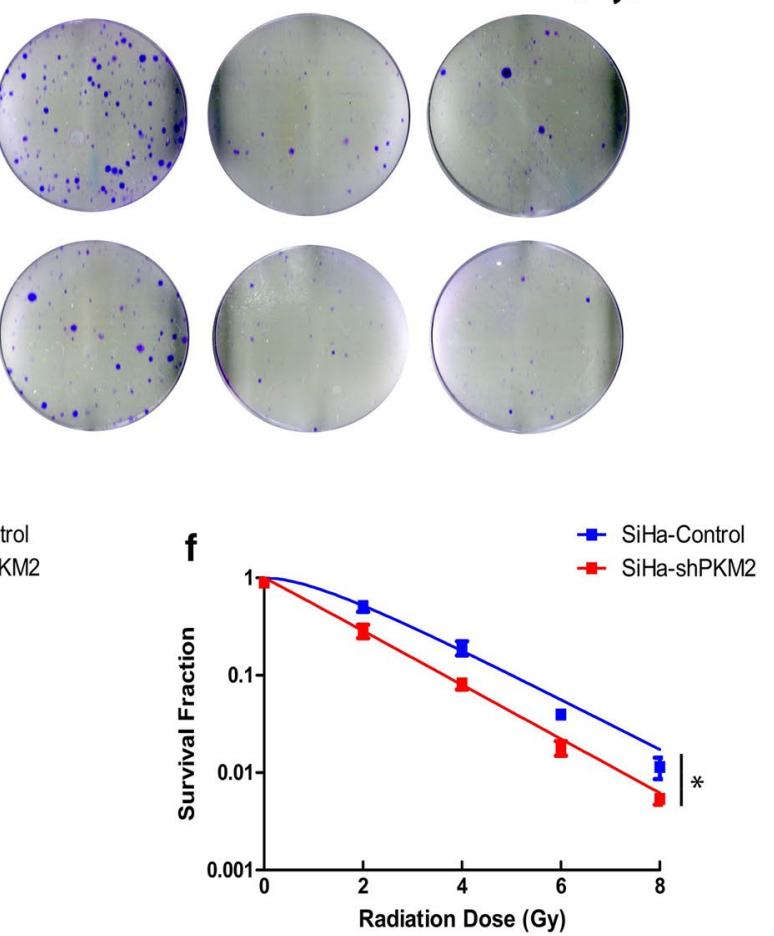

Fig. 2 Knockdown of PKM2 expression enhanced the radiosensitivity of HeLa and SiHa cells in vitro. $\mathbf{a}$, b Cells were irradiated at different doses of irradiation, and the CCK-8 assay was used to test the viability of these cells. $\mathbf{c}$, $\mathbf{d}$ Radiation sensitivity was tested using colony formation assays. Knockdown of PKM2 decreased clonogenic formation in HeLa and SiHa cells compared with control. e, f Survival curves were obtained using a multitarget click mathematical model. Data represent three independent experiments. ${ }^{*} P<0.05,{ }^{* * *} P<0.001$ 

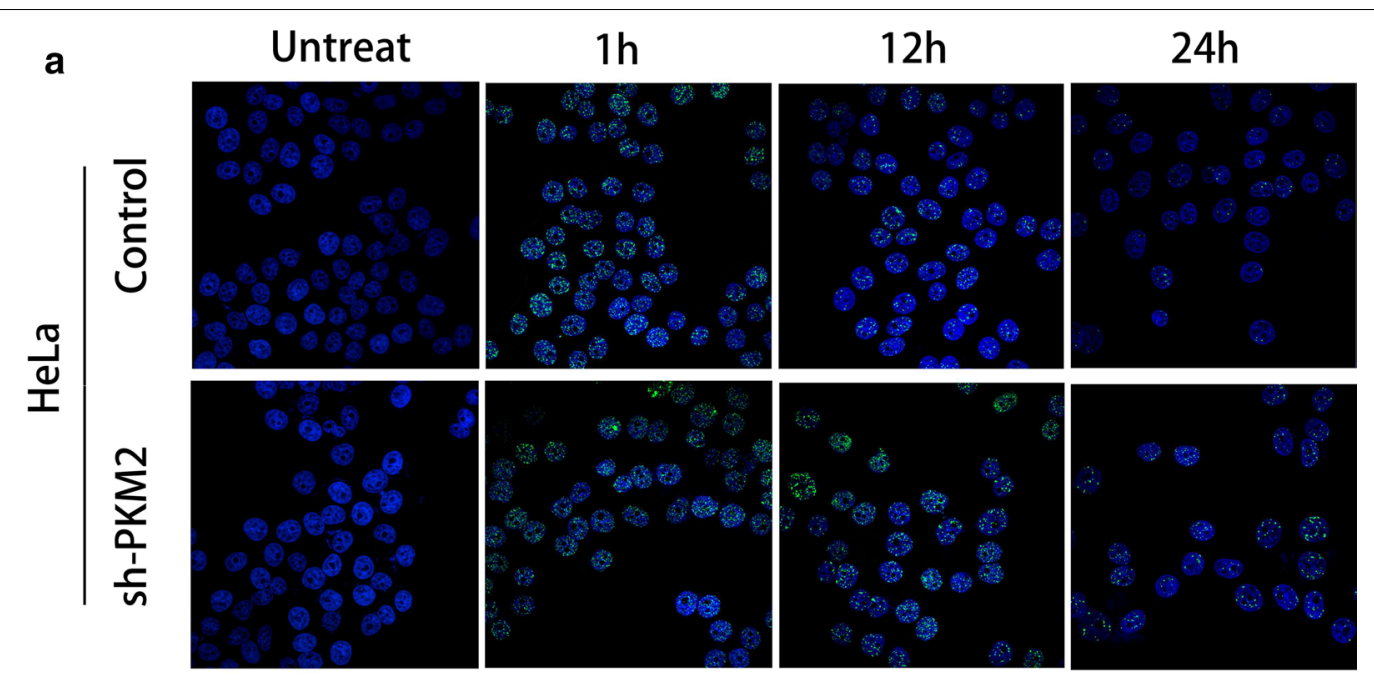

b Untreat

$1 \mathrm{~h}$

$12 \mathrm{~h}$

$24 \mathrm{~h}$
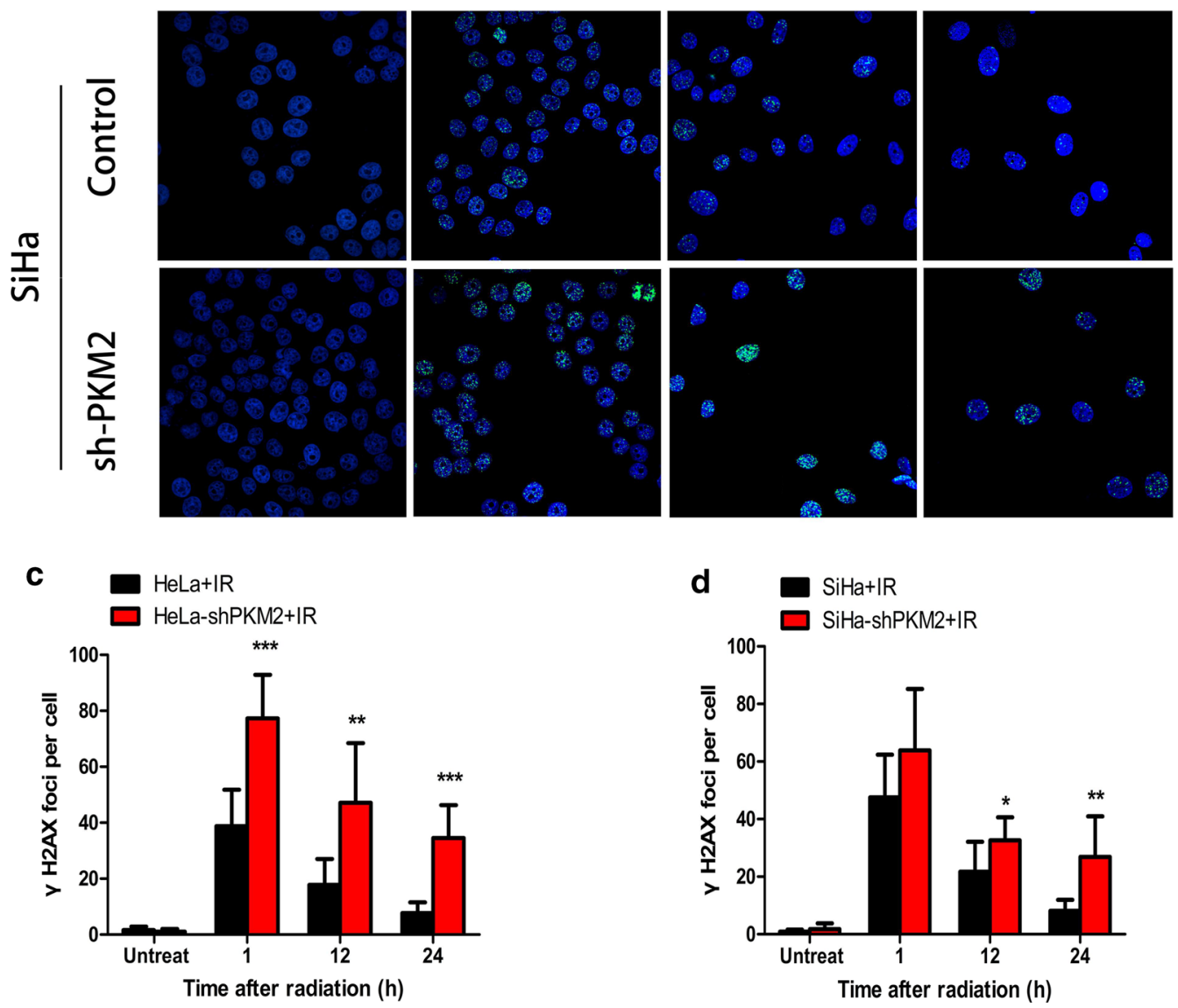

Fig. 3 PKM2 influenced DNA damage repair. a, b Different group of cells was subjected to IR. 1, 12, and $24 \mathrm{~h}$ after IR, cells were fixed and immunostained for $\mathrm{Y}-\mathrm{H} 2 \mathrm{AX}$ foci. The numbers of $\mathrm{Y}-\mathrm{H} 2 \mathrm{AX}$ foci at all time points were measured in both HeLa and SiHa cells. Untreated cells were used as the negative control. Representative images are presented here $(400 \times)$. c, $\mathbf{d}$ Knockdown of PKM2 significantly increased the numbers of $\gamma$ - H2AX foci after different doses of irradiation in both HeLa and SiHa cells. Data represent three independent experiments. ${ }^{*} P<0.05$; ${ }^{* *} P<0.01$; ***P $<0.001$ 


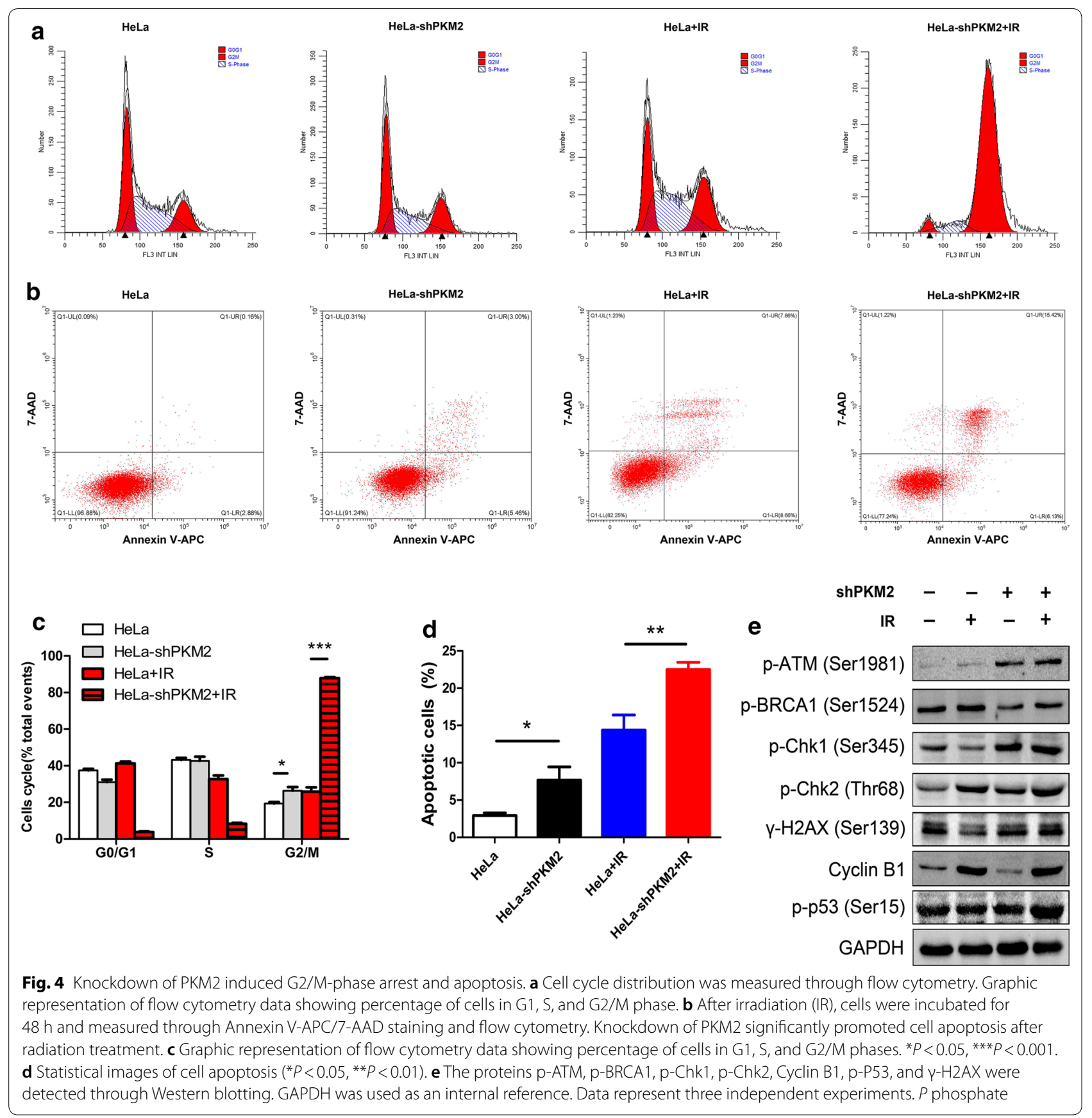

cells were also significantly reduced compared with the expression levels in the controls after irradiation (Fig. 5b). These results indicated that depletion of PKM2 leads to reduced expression of CSC biomarkers.

\section{Discussion}

Radiation therapy has become a vital tool for LACC treatment, but most patients develop local recurrence within 5 years of radiotherapy due to the acquisition of radioresistance [19]. To improve the efficacy of radiotherapy, research that focuses on tumor markers of radiosensitivity has become a major area of development in the field. We evaluated whether PKM2 is a radiosensitivity marker that modulates the response to IR. Remarkably, we found that knockdown of PKM2 inhibited cell growth, increased DNA damage, led to G2/M cell cycle arrest, accompanied by activation of $\mathrm{p} 53$, reduced expression of CSC markers, and thereby enhanced radiosensitivity. 

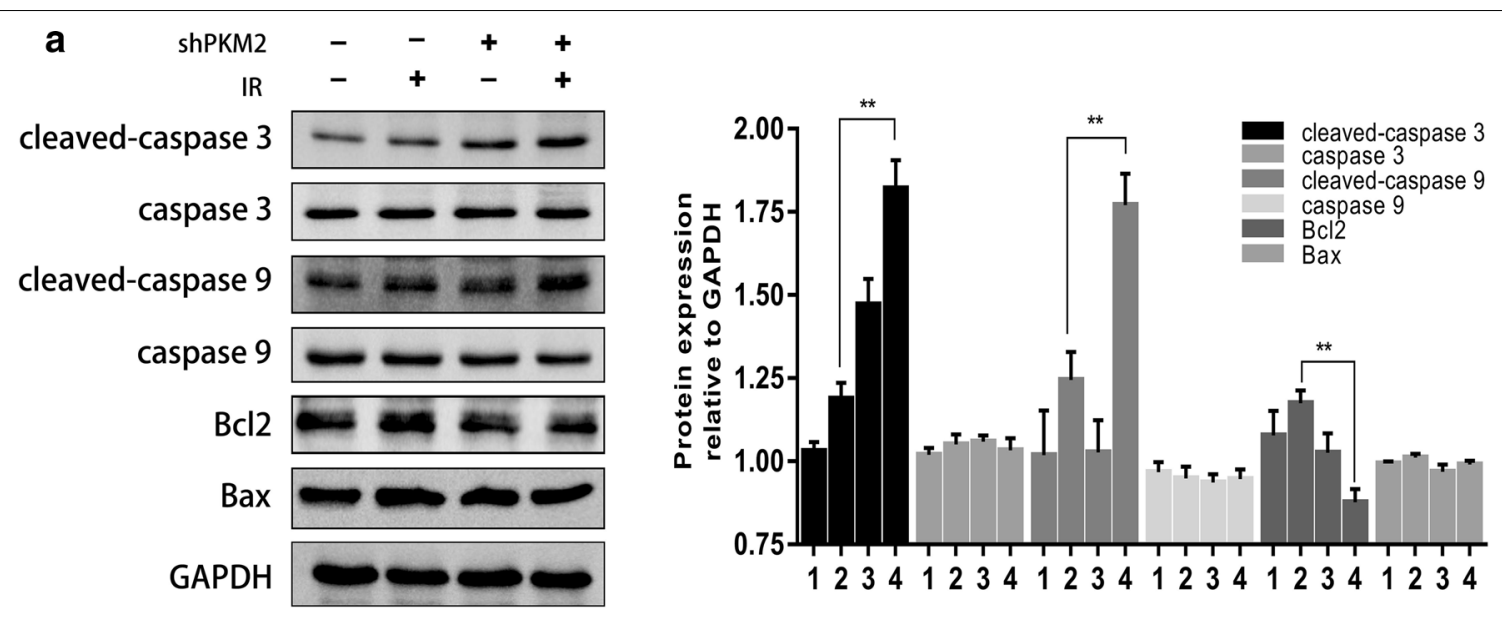

b
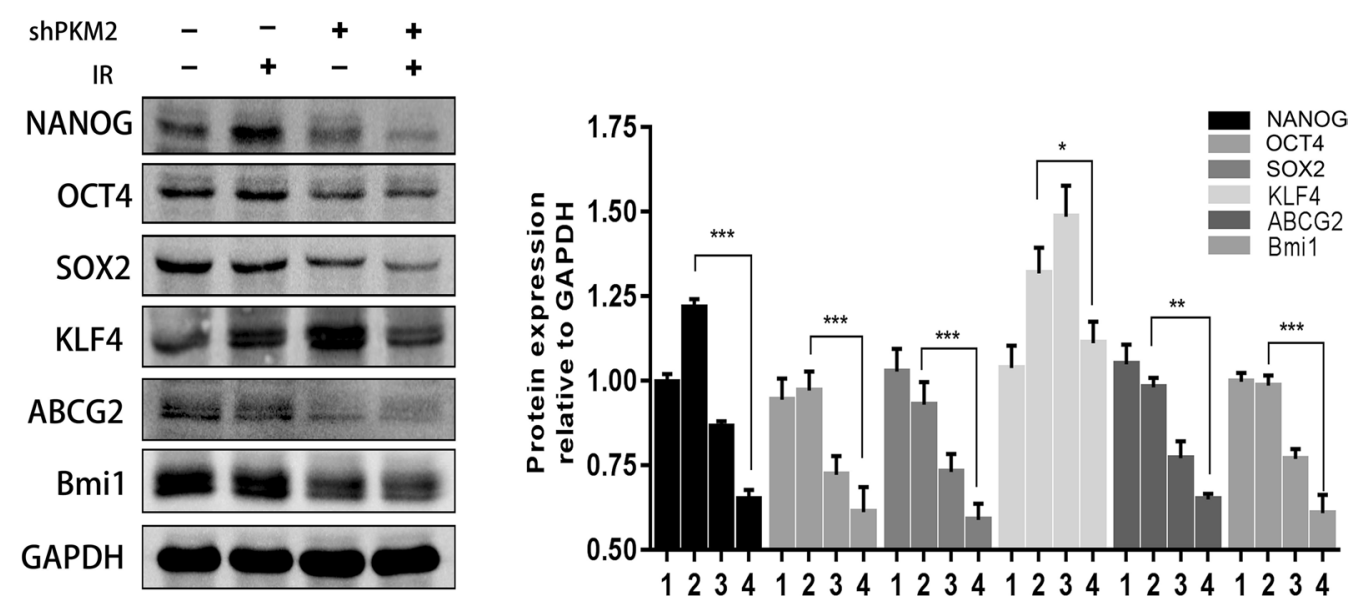

Fig. 5 Western blot for the detection of stemness-associated markers. a Cells were pretreated with or without 2 Gy of IR and were analyzed for the expression of cleaved caspase 3, cleaved caspase 9, caspase 3, caspase 9, Bax, and Bcl2 protein levels through Western blotting. b Knockdown of PKM2 combined with IR downregulated NANOG/OCT4/SOX2 in protein expression. GAPDH protein was used as an internal standard. Data represent three independent experiments. ${ }^{*} P<0.05 ;{ }^{* *} P<0.01$; ${ }^{* *} P<0.001$. (shPKM2: shRNA against PKM2; IR: irradiation. $1=$ control cells, $2=$ control cells $+\mathrm{IR}, 3=$ shPKM2 cells, $4=$ shPKM2 cells $+\mid \mathrm{R})$

The Warburg effect is a common phenomenon in most cancer cells that supports tumor cell growth, even in the presence of ample $\mathrm{O}_{2}$ [20]. Research studies have increasingly found that the Warburg effect is implicated in radiation as well as chemotherapy resistance [21,22]. A previous study [23] revealed that cancer cells recovering from damage undergo mitochondrial restructuring and show increased aerobic glycolysis. Increasing evidence suggests that PKM2 plays a critical role in aerobic glycolysis and that knockdown of PKM2 in cancer cells reduces glucose uptake, increases oxygen consumption, and reduces lactate production to suppress the Warburg effect $[24,25]$. The role of PKM2 in various cancers has been previously investigated [26], but its function in CC has not been fully elucidated. We previously reported that PKM2 is upregulated and suggested that it functions as a tumor promoter in human CC [12]. More importantly, it has been found that downregulation of PKM2 effectively enhances radiosensitivity in human non-small-cell lung carcinoma [10, 27] and glioblastoma multiforme cell lines (U87, T98G, and U251) [28]. As anticipated, our results showed that high PKM2 expression was related to clinical radioresistance in patients with CC. Additionally, exposure of cells to IR increased PKM2 expression suggested that elevated PKM2 expression might contribute to radiation resistance. Moreover, PKM2 inhibition enhanced radiosensitivity of $\mathrm{CC}$ in vitro through inhibition the of survival rate and induction of G2/M arrest followed by radiation-induced apoptosis. 
The mechanism of radiation resistance is complex, and the underlying mechanism of the direct association between PKM2 expression and radiation resistance is not fully understood. A crucial effect of ionizing radiation is the alteration of cell cycle progression, resulting in cell cycle arrest in either G1, S, or the G2/M phases, and the fact that cells in different phases exhibit different radiosensitivity [29]. Cells in the M and G2 phases are the most radiosensitive, those in the G1 phase are less sensitive, and S-phase cells are the most resistant to IR [30]. IR-induced DNA damage results in the activation of the DNA repair mechanism, which is one of the most influential biological processes contributing to radioresistance. H2AX is phosphorylated at the sites of DNA DSBs induced by ionizing radiation and is required for recruitment of repair factors into the nuclear foci after DNA damage [31]. Accordingly, the function of H2AX is believed to be associated primarily with DNA damage. ATM is a key protein kinase which plays a crucial role in the cellular response to IR-induced DNA damage [32]. Checkpoint kinases 1 and 2 (Chk1 and Chk2) have emerged as critical mediators in cell cycle checkpoint control and facilitate cell cycle arrest. Previous studies have shown that activation of the checkpoint kinases results in accumulation of p53, which subsequently modulates the transcription of many proapoptotic genes, thus regulating IR-induced apoptosis [33-35]. In this study, we found that knockdown of PKM2 increases the phosphorylation of ATM, and Chk1 and induces G2/M cell cycle arrest in CC cells. Sizemore et al. [28] also found that ATM phosphorylates PKM2 at T328, and directly regulates DSB repair to cause radiation resistance. However, another study demonstrated that PKM2 interferes with the kinase activity of ATM toward P53 through a potential direct interaction [36]. Therefore, more research is required to clarify the how PKM2 regulates ATM and Chk1 activation. We also found that PKM2 inhibition induced a significant G2/M cell cycle arrest and activated p-p53 expression in irradiated CC cells, indicating that DNA damage was not repaired and culminates in apoptotic cell death. In addition, PKM2 induced caspase 3 -independent apoptosis of CC cells. The Bcl-2 family of proteins, including proapoptotic and antiapoptotic members, is the most influential mediator of cell apoptosis. Our results also indicated that $\mathrm{Bcl}-2$ expression decreases after IR. Thus, PKM2 knockdown promotes IR-induced apoptosis by regulating $\mathrm{Bcl}-2$ and caspase 3 expression, leading to enhanced radiosensitivity.

The existence of CSCs has been implicated in cancer recurrence, which results in cancer treatment failures. Certain proteins, such as OCT4, SOX2, NANOG, and KLF4, are crucial transcription factors for the maintenance of stemness. It has been reported that radioresistance of CC is associated with CSCs [37]. A previous study found that PKM2 is vital for maintaining stem-cell-like properties [38]. Moreover, EGFR has been found to directly interact with PKM2 to regulate the transcription of stemness-related genes and promote the stem-like phenotype, thereby promoting invasion and metastasis [14]. Lee et al. [39] reported that PKM2 modulates the OCT4-dependent transactivation. Among the CSC markers, depletion of NANOG alone is sufficient to reduce the proportion of CSCs. OCT4 and SOX2 are important transcriptional factors, and their expression has been reported to correlate with tumorigenesis, chemoresistance, and maintenance of the stem-cell-like phenotype in cancer cells [40-42], including CC cells [43]. High expression of SOX2 and OCT4 indicates radiation resistance in cervical squamous-cell carcinoma [44]. We also found that knockdown of PKM2 in CC cells reduced the expression of the CSC-related transcription factors NANOG, SOX2, OCT4, Bmi1, and KLF4.

\section{Conclusions}

In summary, knockdown of PKM2 enhanced radiation sensitivity in CC cells by promoting cell apoptosis, inducing cell cycle arrest at the G2/M phase, thereby increasing radiation-induced DSBs. Such a mechanism may be used to reduce expression of stemness-related genes in CC cells. The results of this study indicate that targeting PKM2 may be a novel potential therapeutic option to increase the radiosensitivity of $\mathrm{CC}$.

\section{Additional files}

Additional file 1: Table S1. Patient characteristics

Additional file 2: Figure S1. Knockdown of PKM2 induces a G2/M cell cycle arrest and apoptosis in SiHa cells. (A) Cell cycle distribution was measured through flow cytometry. Graphic representation of flow cytometry data showing percentage of cells in G1, S, and G2/M. (B) Knockdown of PKM2 significantly promoted cell apoptosis after radiation treatment in SiHa cells. (C) Graphic representation of flow cytometry data showing percentage of cells in G1, S, and G2/M phases. ${ }^{*} P<0.05$, ${ }^{* *} P<0.01$. (D) Statistical image of cell apoptosis, ${ }^{*} P<0.05 ;{ }^{* *} P<0.01 ;{ }^{* * *} P<0.001$.

\section{Abbreviations}

PKM2: pyruvate kinase isozyme type M2; CC: cervical cancer; LACC: locoregionally advanced CC; PEP: phosphoenolpyruvate; CSCs: cancer stem cells; CR: complete response; IR: ionizing radiation; IHC: immunohistochemistry; nCR: noncomplete response; PE: plating efficiency; SF: surviving fraction; SD: standard deviation; DSBs: double-strand DNA breaks; Chk1: checkpoint kinases 1; Chk2: checkpoint kinases 2.

\section{Acknowledgements}

The authenticity of this article was validated by uploading the key raw data into the Research Data Deposit public platform (www.researchdata.org.cn) with the approval number RDDB2019000545. 


\section{Authors' contributions}

XC conceived of, designed, and supervised the study. YL, HZ, YO and ZL performed the experiments. CC analyzed the data. QH provided technical assistance with the experiments. $X C$ and $Y L$ wrote the manuscript. All co-authors have reviewed this version of the manuscript. All authors read and approved the final manuscript

\section{Funding}

This work was supported by the Guangdong Natural Science Foundation (2017A030310197).

\section{Availability of data and materials}

All data generated or analysed during this study are included in this published article and its Additional files.

\section{Ethics approval and consent to participate}

The collection of human tissue samples was approved and supervised by the Ethics Committee of Sun Yat-sen University. All patients provided written informed consent.

\section{Consent for publication}

Not applicable.

\section{Competing interests}

The authors declare that they have no competing interests.

\section{Author details}

${ }^{1}$ Department of Radiation Oncology, Sun Yat-sen University Cancer Center, State Key Laboratory of Oncology in South China, Collaborative Innovation Center for Cancer Medicine, Guangzhou, China. ${ }^{2}$ Gynecology Department, Jinan Maternity and Child Care Hospital, Jinan, China. ${ }^{3}$ Department of Oral and Maxillofacial Surgery, The First Affiliated Hospital, Sun Yat-Sen University, Guangzhou, China. ${ }^{4}$ Department of Pathology, Sun Yat-sen University Cancer Center, State Key Laboratory of Oncology in South China, Collaborative Innovation Center for Cancer Medicine, Guangzhou, China.

Received: 20 January 2019 Accepted: 30 April 2019

Published online: 14 May 2019

\section{References}

1. Sawaya GF. Cervical cancer screening-moving from the value of evidence to the evidence of value. JAMA Intern Med. 2018:178(10):1293-5.

2. Lin Y, Chen K, Lu Z, Zhao L, Tao Y, Ouyang Y, Cao X. Intensity-modulated radiation therapy for definitive treatment of cervical cancer: a metaanalysis. Radiat Oncol. 2018;13(1):177.

3. Schwickert G, Walenta S, Sundfor K, Rofstad EK, Mueller-Klieser W. Correlation of high lactate levels in human cervical cancer with incidence of metastasis. Cancer Res. 1995;55(21):4757-9.

4. Walenta S, Salameh A, Lyng H, Evensen JF, Mitze M, Rofstad EK, MuellerKlieser W. Correlation of high lactate levels in head and neck tumors with incidence of metastasis. Am J Pathol. 1997;150(2):409-15.

5. Shimura T, Noma N, Sano Y, Ochiai Y, Oikawa T, Fukumoto M, Kunugita N. AKT-mediated enhanced aerobic glycolysis causes acquired radioresistance by human tumor cells. Radiother Oncol. 2014;112(2):302-7.

6. Sattler UG, Meyer SS, Quennet V, Hoerner C, Knoerzer H, Fabian C, Yaromina A, Zips D, Walenta S, Baumann M, et al. Glycolytic metabolism and tumour response to fractionated irradiation. Radiother Oncol. 2010;94(1):102-9.

7. Quennet V, Yaromina A, Zips D, Rosner A, Walenta S, Baumann M, MuellerKlieser W. Tumor lactate content predicts for response to fractionated irradiation of human squamous cell carcinomas in nude mice. Radiother Oncol. 2006;81(2):130-5.

8. Son JY, Yoon S, Tae IH, Park YJ, De U, Jeon Y, Park YJ, Rhyu IJ, Lee BM, Chung $\mathrm{KH}$, et al. Novel therapeutic roles of MC-4 in combination with everolimus against advanced renal cell carcinoma by dual targeting of Akt/pyruvate kinase muscle isozyme $\mathrm{M} 2$ and mechanistic target of rapamycin complex 1 pathways. Cancer Med. 2018;7(10):5083-95.

9. Wang HJ, Pochampalli M, Wang LY, Zou JX, Li PS, Hsu SC, Wang BJ, Huang SH, Yang P, Yang JC, et al. KDM8/JMJD5 as a dual coactivator of AR and
PKM2 integrates AR/EZH2 network and tumor metabolism in CRPC. Oncogene. 2018;38(1):17-32.

10. Meng MB, Wang HH, Guo WH, Wu ZQ, Zeng XL, Zaorsky NG, Shi HS, Qian D, Niu ZM, Jiang B, et al. Targeting pyruvate kinase M2 contributes to radiosensitivity of non-small cell lung cancer cells in vitro and in vivo. Cancer Lett. 2015;356(2 Pt B):985-93.

11. Zhao Y, Shen L, Chen X, Qian Y, Zhou Q, Wang Y, Li K, Liu M, Zhang S, Huang $X$. High expression of PKM2 as a poor prognosis indicator is associated with radiation resistance in cervical cancer. Histol Histopathol. 2015;30(11):1313-20

12. Lin Y, Meng F, Lu Z, Chen K, Tao Y, Ouyang Y, Cao X. Knockdown of PKM2 suppresses tumor progression in human cervical cancer by modulating epithelial-mesenchymal transition via Wnt/beta-catenin signaling. Cancer Manag Res. 2018;10:4191-202.

13. Yan B, Liu S, Shi Y, Liu N, Chen L, Wang X, Xiao D, Liu X, Mao C, Jiang Y, et al. Activation of AhR with nuclear IKKalpha regulates cancer stemlike properties in the occurrence of radioresistance. Cell Death Dis. 2018;9(5):490.

14. Shi Y, Liu N, Lai W, Yan B, Chen L, Liu S, Liu S, Wang X, Xiao D, Liu X, et al. Nuclear EGFR-PKM2 axis induces cancer stem cell-like characteristics in irradiation-resistant cells. Cancer Lett. 2018;422:81-93.

15. Christensen DR, Calder PC, Houghton FD. GLUT3 and PKM2 regulate OCT4 expression and support the hypoxic culture of human embryonic stem cells. Sci Rep. 2015:5:17500.

16. Lee J, Kim HK, Han YM, Kim J. Pyruvate kinase isozyme type M2 (PKM2) interacts and cooperates with Oct-4 in regulating transcription. Int J Biochem Cell Biol. 2008;40(5):1043-54.

17. Heery CR, O'Sullivan-Coyne G, Madan RA, Cordes L, Rajan A, Rauckhorst M, Lamping E, Oyelakin I, Marte JL, Lepone LM, et al. Avelumab for metastatic or locally advanced previously treated solid tumours (JAVELIN Solid Tumor): a phase 1a, multicohort, dose-escalation trial. Lancet Oncol. 2017;18(5):587-98.

18. Parris CN, Adam ZS, Al-Ali H, Bourton EC, Plowman C, Plowman PN. Enhanced gamma-H2AX DNA damage foci detection using multimagnification and extended depth of field in imaging flow cytometry. Cytometry A. 2015;87(8):717-23.

19. Xiong H, Nie X, Zou Y, Gong C, Li Y, Wu H, Qiu H, Yang L, Zhuang L, Zhang $P$, et al. Twist1 enhances hypoxia induced radioresistance in cervical cancer cells by promoting nuclear EGFR localization. J Cancer. 2017:8(3):345-53.

20. Li Q, Wei P, Wu J, Zhang M, Li G, Li Y, Xu Y, Li X, Xie D, Cai S, et al. The FOXC1/FBP1 signaling axis promotes colorectal cancer proliferation by enhancing the Warburg effect. Oncogene. 2018;38(4):483.

21. Pitroda SP, Wakim BT, Sood RF, Beveridge MG, Beckett MA, MacDermed DM, Weichselbaum RR, Khodarev NN. STAT1-dependent expression of energy metabolic pathways links tumour growth and radioresistance to the Warburg effect. BMC Med. 2009;7:68.

22. Khodarev NN, Beckett M, Labay E, Darga T, Roizman B, Weichselbaum RR. STAT1 is overexpressed in tumors selected for radioresistance and confers protection from radiation in transduced sensitive cells. Proc Natl Acad Sci USA. 2004;101(6):1714-9.

23. Akakura S, Ostrakhovitch E, Sanokawa-Akakura R, Tabibzadeh S. Cancer cells recovering from damage exhibit mitochondrial restructuring and increased aerobic glycolysis. Biochem Biophys Res Commun. 2014;448(4):461-6.

24. Christofk HR, Vander HM, Harris MH, Ramanathan A, Gerszten RE, Wei R, Fleming MD, Schreiber SL, Cantley LC. The M2 splice isoform of pyruvate kinase is important for cancer metabolism and tumour growth. Nature. 2008;452(7184):230-3.

25. Vander HM, Christofk HR, Schuman E, Subtelny AO, Sharfi H, Harlow EE, Xian J, Cantley LC. Identification of small molecule inhibitors of pyruvate kinase M2. Biochem Pharmacol. 2010;79(8):1118-24.

26. Li YH, Li XF, Liu JT, Wang H, Fan LL, Li J, Sun GP. PKM2, a potential target for regulating cancer. Gene. 2018;668:48-53.

27. Wang S, Ma Y, Wang P, Song Z, Liu B, Sun X, Zhang H, Yu J. Knockdown of PKM2 enhances radiosensitivity of non-small cell lung cancer. Cell Biochem Biophys. 2015;73(1):21-6.

28. Sizemore ST, Zhang M, Cho JH, Sizemore GM, Hurwitz B, Kaur B, Lehman NL, Ostrowski MC, Robe PA, Miao W, et al. Pyruvate kinase M2 regulates homologous recombination-mediated DNA double-strand break repair. Cell Res. 2018;28(11):1090-102. 
29. Yu X, Xiao H, Zhao B, Zhang X, Wang G. DNA repair gene ERCC1 C118T polymorphism predicts sensitivity of recurrent esophageal cancer to radiochemotherapy in a Chinese population. Thorac Cancer. 2015;6(6):741-8.

30. Chetty C, Bhoopathi P, Rao JS, Lakka SS. Inhibition of matrix metalloproteinase-2 enhances radiosensitivity by abrogating radiation-induced FoxM1-mediated G2/M arrest in A549 lung cancer cells. Int J Cancer. 2009;124(10):2468-77.

31. Nowsheen S, Aziz K, Luo K, Deng M, Qin B, Yuan J, Jeganathan KB, Yu J, Zhang H, Ding W, et al. ZNF506-dependent positive feedback loop regulates H2AX signaling after DNA damage. Nat Commun. 2018;9(1):2736.

32. Dhume K, McKinstry KK. Early programming and late-acting checkpoints governing the development of CD4 T-cell memory. Immunology. 2018:155(1):53-62.

33. Meng X, Bi J, Li Y, Yang S, Zhang Y, Li M, Liu H, Li Y, Mcdonald ME, Thiel KW, et al. AZD1775 increases sensitivity to olaparib and gemcitabine in cancer cells with p53 mutations. Cancers (Basel). 2018;10(5):149.

34. Iwasa H, Sarkar A, Shimizu T, Sawada T, Hossain S, Xu X, Maruyama J, Arimoto-Matsuzaki K, Withanage K, Nakagawa K, et al. UNC119 is a binding partner of tumor suppressor Ras-association domain family 6 and induces apoptosis and cell cycle arrest by MDM2 and p53. Cancer Sci. 2018;109(9):2767-80.

35. Spiegelberg D, Mortensen AC, Lundsten S, Brown CJ, Lane DP, Nestor M. The MDM2/MDMX-p53 antagonist PM2 radiosensitizes wild-type p53 tumors. Cancer Res. 2018;78(17):5084-93.

36. Xia L, Wang XR, Wang XL, Liu SH, Ding XW, Chen GQ, Lu Y. A novel role for pyruvate kinase $\mathrm{M} 2$ as a corepressor for p53 during the DNA damage response in human tumor cells. J Biol Chem. 2016;291(50):26138-50.

37. Lopez J, Poitevin A, Mendoza-Martinez V, Perez-Plasencia C, Garcia-Carranca A. Cancer-initiating cells derived from established cervical cell lines exhibit stem-cell markers and increased radioresistance. BMC Cancer. 2012;12:48
38. Yang YC, Chien MH, Liu HY, Chang YC, Chen CK, Lee WJ, Kuo TC, Hsiao M, Hua KT, Cheng TY. Nuclear translocation of PKM2/AMPK complex sustains cancer stem cell populations under glucose restriction stress. Cancer Lett. 2018;421:28-40.

39. Deng L, Xiang X, Yang F, Xiao D, Liu K, Chen Z, Zhang R, Feng G. Functional evidence that the self-renewal gene NANOG regulates esophageal squamous cancer development. Biochem Biophys Res Commun. 2017;490(2):161-8.

40. Singh S, Trevino J, Bora-Singhal N, Coppola D, Haura E, Altiok S, Chellappan SP. EGFR/Src/Akt signaling modulates Sox2 expression and selfrenewal of stem-like side-population cells in non-small cell lung cancer. Mol Cancer. 2012;11:73.

41. Takahashi K, Yamanaka S. Induction of pluripotent stem cells from mouse embryonic and adult fibroblast cultures by defined factors. Cell. 2006;126(4):663-76.

42. Bayo P, Jou A, Stenzinger A, Shao C, Gross M, Jensen A, Grabe N, Mende $\mathrm{CH}$, Rados PV, Debus J, et al. Loss of SOX2 expression induces cell motility via vimentin up-regulation and is an unfavorable risk factor for survival of head and neck squamous cell carcinoma. Mol Oncol. 2015;9(8):1704-19.

43. Kim BW, Cho H, Choi CH, Ylaya K, Chung JY, Kim JH, Hewitt SM. Clinical significance of OCT4 and SOX2 protein expression in cervical cancer. BMC Cancer. 2015;15:1015.

44. Shen L, Huang X, Xie X, Su J, Yuan J, Chen X. High expression of SOX2 and OCT4 indicates radiation resistance and an independent negative prognosis in cervical squamous cell carcinoma. J Histochem Cytochem. 2014;62(7):499-509.

\section{Publisher's Note}

Springer Nature remains neutral with regard to jurisdictional claims in published maps and institutional affiliations.
Ready to submit your research? Choose BMC and benefit from:

- fast, convenient online submission

- thorough peer review by experienced researchers in your field

- rapid publication on acceptance

- support for research data, including large and complex data types

- gold Open Access which fosters wider collaboration and increased citations

- maximum visibility for your research: over $100 \mathrm{M}$ website views per year

At BMC, research is always in progress.

Learn more biomedcentral.com/submissions 